\title{
An Effective Technique for Tuning the Time Delay System with PID Controller-Ant Lion Optimizer Algorithm with ANN Technique
}

\author{
Thomas George, V. Ganesan
}

\begin{abstract}
Nowadays, the PID controller is very common controller as well as very important controller in industrial utilizations. In the paper, proposed an ALO algorithm and ANN controller is utilized to enhance PID controller performance and control the tuning of TDS. TDS stands for Time delay system. ALO stands for Ant lion optimizer and ANN stands for Artificial neural network. In terms of parameters controlling, the time delay system is controlled and for different delay events low overshoot and fast time settling is reached. The novelty of the presented method is enhancing the PID controller performance by optimizing the PID gain parameters and controlling the highorder TDS. The performance of time delay system can be enhanced through decreasing error, tracking, time delay \& error, rapid and exactly for their corresponding reference values. For parameter controlling of time delay system along optimal values, can be significantly enhanced the performance. To analyze the characteristics of the presented method, the various time delay systems are analyzed. The input and gain parameters were utilized to evaluate the objective function from tuning system. Based on proposed method, the optimal result is achieved and evaluated the increae time, settling time, overshoot as well as steady state error in TDS. The suggested controller is executed in MATLAB/Simulink work site and the presented technique performance examined through performance indexes and time domain specifications are evaluated using presented method compared to previous methods like ABC (Artificial Bee colony) algorithm, GSA (Gravitational Search Algorithm) ,FA (Firefly Algorithm).
\end{abstract}

Keywords: PID Controller, Time Delay System, Optimal values, parameters.

\section{INTRODUCTION}

Many industrial processes consist of high- order dynamics along time delay as well as nonlinear character over known disturbances or unknown disturbances [1]. TDS meets in different regions, along engineering, biology field and economics. TDS is commonly a step toward instability as well as variations in the methods [2]. TDS are aftereffect or dead-time, belonging to FDEs (functional differential equations) class, in contrast to ODEs (ordinary differential equations) [3]. Because of inherent nonlinearity, most

Revised Manuscript Received on February 05, 2020.

* Correspondence Author

Thomas George*, Research Scholar, Dept of Electronics \& Communication, Sathyabama University Chennai, India.j4jiju@gmail.com

Dr. V. Ganesan, Assistant Professor, Dept of Electronics \& Communication, Sathyabama University Chennai, India. vganesh1711@gmail.com

(C) The Authors. Published by Blue Eyes Intelligence Engineering and Sciences Publication (BEIESP). This is an open access article under the CC BY-NC-ND license (http://creativecommons.org/licenses/by-nc-nd/4.0/)

chemical process industries require modern control techniques [4]. Although, applying high-order process approximation through low-order model with delay of time provides simplifying control algorithms. Using standard feedback controls processes with time delay are difficult to control [5]. However, several improved control methods has created, PID controller is very famous in industrial field because of its simplicity, compatibility and robustness [6, 7].

In spite of their successful to the system of linear, usual PID controller is unfit to the system of non-linear and highorder TDS, does not refer to intricate and ambiguous systems which need skilled knowledge [8]. In industrial utilizations PID controller is mostly applied. In the PID controller parameters including integral gain, proportional gain, and derivative gain, could develop the performance of system [9]. A closed-loop system has lot of examples, like direction control, level control, temperature control, robot mechanism control and so on [10]. A closed-loop system may be unstable due to small disturbances in the values of the designed control parameters [11]. Further, methods for changing the PID controller parameters have been increasing over the past few decades [12]. Some another kind of methods are theoretical calculations method, classical Z-N (Ziegler-Nichols) method, pole placement and its related Cohen-Coon method and IMC (internal model control) method. The nonlinear system model is difficult to identify accurately [13]. Z-N method is the oldest method as well as simplest method. Z-N method supplies a big overshoot and settling time. So, PID parameters values are often refined subsequently as per operator's experience [14]. In addition, IMC based PID controllers are also considered to derive set-point tracking benchmarks [15]. One of the advanced control strategies are IMC that is well power, easy design and tune. Even though, conventional IMC does not apply to transient process or TDS [16]. PID controllers tuning rules reflect an increase in interest in PID controllers use. Evolutionary optimization techniques other than routine tuning methods like GA, PSO and FF algorithm. GA stands for Genetic Algorithm, PSO stands for Particle swarm optimization and FF stands as Firefly [17]. Bacterial Foraging (BF) [18], ACO (Ant Colony Optimization) [19] are commonly applied to developing parameter controller [20]. Here, performance of ALO algorithm and ANN technique is utilized for enhancing PID controller performance and control tuning of time delay system.

In this study, the remaining sections are designed given below. The recent research works on protection scheme in DG is explained in section 2.

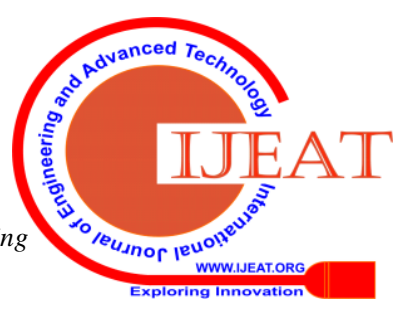




\section{An Effective Technique for Tuning the Time Delay System with PID Controller-Ant Lion Optimizer Algorithm with ANN Technique}

In section 3 formulated the system description and the presented scheme. The experimental results are defined in section 4. Finally, concluded proposed adaptive protection scheme in DG.

\section{RECENT RELATED WORKS: AN OVERVIEW}

Numbers of research work have previously been in the literature, based on the PID controller performance with the TDS. Here, some works were reviewed.

K.Ghousiya Begum et al. [21] have proposed an IMC (internal model control) with the optimal $\mathrm{H}_{2}$ reducing framework to PID controllers design. The design of controller was labeled to integrate and double integrate process on TDS along RHP zeros. RHP stands for Right half plane. To obtain optimal controller the Blaschke product was utilized. For the designing of controller tuning parameter of single adjustable closed loop was used. To select tuning parameter systematic guidelines based on maximum sensitivity were presented.

PipatPrommeeet al. [22] was proposed a PID controller log-domain whereas the bias currents were used to the PID components to individualistic or at same time electronic tuning and response of following enhanced. Initial PID circuits 17, 13 and 21 were realized separately using bipolar transistor, and need 62 bipolar transistors for final PID controller, multi-output current buffer and biasing circuits. The presented PID controller operates at a power on $\pm 1.5 \mathrm{~V}$ dual power, without the circuit topology change along widerange turn-ability up to three decades.

Qiu Han Seer et al. [23] have presented stabilization through second order unstable processes PID controller. The PID controller specified as SODUP and SODTUP which was operated through essential and enough basis on the analysis of Routh-Hurwitz stability. SODUP and SODTUP stands for second-order dead time along an unstable pole and second-order dead time with two unstable poles respectively. Examines of stability presents enhanced of existence understanding on each PID parameter had stabilizing range. Three tuning algorithms of PID were presented for given wanted closed-loop performancerobustness including stable regions controller parameters received through stability analysis.

Anupam Kumar et al. [24] have proposed a concept of IT2FO-FPID controller that needs fractional order integrator and differentiator fractional order. IT2FO-FPID stands for interval type-2 fractional order fuzzy PID. The combination of TSK type IT2FLC with PID-type fractional controller was researched for measuring time response owing to unit step response and disturbance of unit load. TSK stands for Takagi-Sugeno-Kang and IT2FLC stands for interval type-2 fuzzy logic controller. Fractional order the integratordifferentiator operators were assumed like design variables within the scaling factors of input output in order to design this controller. ABC is the hybridized algorithm GA is employed for optimizing parameters controller when decreasing sum of weight on ITAE and ISCO. ITAE stands for Integral time absolute error and ISCO stands for Integral square of control output.

Helem Sabina Sanchez et al. [25] have proposed the procedures of tuning for proportional-integral-derivative

controllers one degree of freedom, through assuming main factors like robustness of control system by regulating service trade-off in efficiency and modes of operational performance and maximum sensitivity peak. To create trade-off optimal solutions different conflicting objectives were handled through utilizing the algorithm named as multi-purpose optimization. A simple tuning rule was defined by applying Nash solutions like the technique of multi-criteria decision-making. Nash basis have been display for present suitable trading results for the problem of controller tuning.

Jerome Mendes et al. [26] have addressed the controlling issues that are unknown to industrial utilizations and time varying plants. To control unknown and time-varying plants the plug-and-play method was followed with used one algorithm named as control algorithm that automatically modify the control parameters. STC tested the actual process structure with the PID type. STC stands for Self Tuning Controllers. The structure had two connected DC motors and a variable load.

Donghyeon Lee et al. [27] have proposed a MCFC (Molten Carbonate Fuel Cell) operation framework in terms of PID auto-tuning control. MCFC was emerging as a promising renewable power system. To meet its varying demands operate the MCFC was more challenging due to its nonlinearity and complex dynamics.

Xiaoli Luan et al. [28] have designed a pre-compensator based transparent multiple time delay processes per single TDS with the path forward. In the method, any wellestablished approach can be used to design the control of input / output time-delay plants. In the feedback path, this method was applied directly along multiple TDS with stable plants, and may expand with unstable plants along few restrictions.

Qinqin Chai et al. [29] have presented a free terminal optimized time control issue that requires determining both the terminal time and control, as the cost function was reduced, including continuous inequality restrictions. To resolve this optimized control issue in the free terminal time, the control parameter method was used to approximate the control function as a static control function in parts that heights as well as switching times were considered as variables of decision. The issue of optimal control of free terminal time was estimated as series of issues of choosing optimal parameters governed to linear time delay systems, everyone could seen as issue of non-linear optimization. A complete technique of particle swarm optimization information was espoused to resolve the approximation issue. At last, two issues of optimized free terminal time control were solved, within the issues of optimal fisheries control, applying the technique presented to demonstrate its applicability.

\section{PROPOSED METHODOLOGY OF PID CONTROLLER}

In General, the high-order plant of process control is approximated to the first or second-order plant with time delay. 
Hence, we labeled our discussion for designing on ALO in terms of ANN-PID controller through approximating high-order model to second-order systems in this presented approach. Figure 1 depicts the presented control process proceeds in the given pattern and on PID controller standard block diagram. To initiate PID gain values the Pole-zero cancellation technique is used. In equation (1) represents mathematically of PID controller,

$$
u(t)=K_{P}\left[e(t)+\frac{1}{T_{i}} \int_{0}^{t} e(\tau) d \tau+T_{d} \frac{d e(t)}{d(t)}\right]
$$

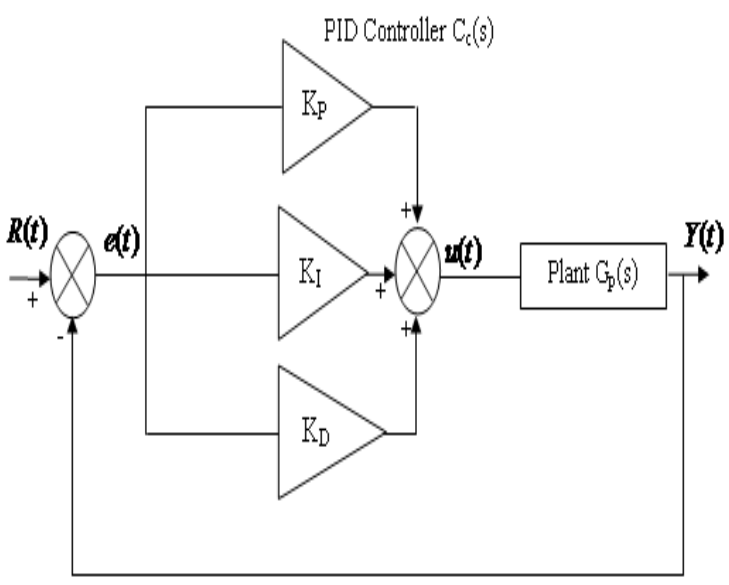

Figure1. PID controller block diagram

Here, the system's control and error signals represents $u(t)$ and $e(t)$. The proportion gain specifies $K_{P}$, the integral and derivative time constants denotes $T_{i}$ and $T_{d}$ [30]. The corresponding PID controller transfer function $G_{c}(s)$ provides the equation (2),

$G_{C}(s)=K_{P}\left[1+\frac{1}{T_{i} s}+T_{d} s\right]$

Here it is obviously $K_{I}=K_{P} / T_{i}$ and $K_{D}=K_{P} T_{d}$ that is rewritten as equation (3),

$G_{C}(s)=u(t)=\left[K_{P}+\frac{K_{I}}{s}+K_{D}\right]$

Here, controller's integral and derivative gain values denoted by $K_{I}$ and $K_{D}$.

\section{A. High-Order Time Delay System}

Assuming $n^{\text {th }}$ order invariant linear time continuous system, which is specified the higher order system as equation (4),
$G_{h}(s)=\frac{N(s)}{D(s)}=\frac{\sum_{k=1}^{n-1} A_{k} s^{k}}{\sum_{k=0}^{n} a_{k} s^{k}}$

Here, $N(s)$ represents polynomial numerator and $D(s)$ represents polynomial denominator. Moreover $A_{k}$ and $a_{k}$ represents constant coefficients on s-terms of numerator and denominator of $G_{h}(s)$ [31]. High-order TDS $G_{p}(s)$ specifies multiplication on the $G_{h}(s)$ as well as time delay $e^{-\theta s}$ that is described in equation (5),

$G_{p}(s)=K \times G_{h}(s) \times e^{-\theta s}$

Here, $K$ is the steady state gain constant. Let us consider $G_{h}(s)$ denotes higher order system and $e^{-\theta s}$ denotes time delay which is combined with PID controller $G_{c}(s)$ [32]. The PID controller is mathematical formulation of the combined system output can be described in the equation (6)

$Y(s)=u(s) \times G_{p}(s)$

For a desired output the important objective on structured on PID controller for gains tuning $K_{P}, K_{I}$ as well as $K_{D}$. To minimize the computational complexities and implementation difficulties, high-order TDS is decreased into lesser the second order system. According to design specification to design the model on second-order controller of PID adjusted. Furthermore, the closed loop response obtained in new low-order model connected to the PID controller, which represents the original high-order TDS response properties along the PID controller.

\section{B. Performance Intex on Proposed Controller}

The structured PID controller is to stabilize the system and present better set point tracking and better interrupt rejection with robustness to uncertainty of parameter. The performance of technique is expressed based ISE, IAE and settling time. ISE stands for Integral square error and IAE stands for Integral absolute error. For comparing PID controller performance, three criterions are used that is (1) performance index (2) stability/robustness and (3) input usage. The given efficiency indices is employed to compare the controller performance structured through presented technique along other recently reported methods [33]. By increasing the following fitness function the proposed technique the optimal solution is searched i.e. given an evaluation function a quality measure on the solution for issues. The ISE penalizes large errors, which is derived as equation (7),

ISE $=\int_{0}^{\infty} e^{2} d t$ 


\section{An Effective Technique for Tuning the Time Delay System with PID Controller-Ant Lion Optimizer Algorithm with ANN Technique}

The objective function is to decrease IAE that defined any industrial process performance at control process. Then the IAE treats all the errors are same and quation (8) is defined as error.

$$
I A E=\int_{0}^{\infty}|e| d t
$$

Finally, the ITAE penalizes long-standing errors, which is provide as equation (9),

$$
\text { ITAE }=\int_{0}^{\infty} t|e| d t
$$

Here, $e$ represents error that is difference among set point and measurement. At current work, the performance measure is used by IAE as several authors in the literature addressed the performance based IAE [34]. Any one smaller value indices indicate that particular technique when compare other methods is performing better.

\section{Proposed Technique to Tuning the PID Parameters}

An adaptive technique for improving the performance index and tuning PID controller gain parameters presented on this paper. In this presented method in terms of ANN multi-layer, trained network to improving value along use of ALO. The proposed technique has ANN there all the neurons are trained to ALO. The ANN is used to generate the gain parameters on PID controller, but training dataset of the ANN is not an optimal level, so we required the optimal dataset. The ALO algorithm is used to select optimal training dataset based on adequate gain parameters on PID controller. Suggested method is described below.

\section{1) Optimal PID parameter search using ANN}

This section describes optimal gain parameter estimation using the ANN technique, which is based on a human learning approach, which models a human brain and has many artificial neurons. The fundamental element of ANN is neurons. Since other neurons the neurons receive input and output is generated through synapse of firing. The neurons make sum of weighted of entire inputs, after produce an output by a transfer function. The ANNs structured into three layers. They are output layer, input layer and hidden layer that illustrated at figure 2. Normally, the use of input layer is receives the collected data, the use of output layer is generates calculated information. The root of ANN is hidden layers that perform the network's actual computations [35]. When a network given specific inputs set it takes effect and the layer of output generates wanted result.

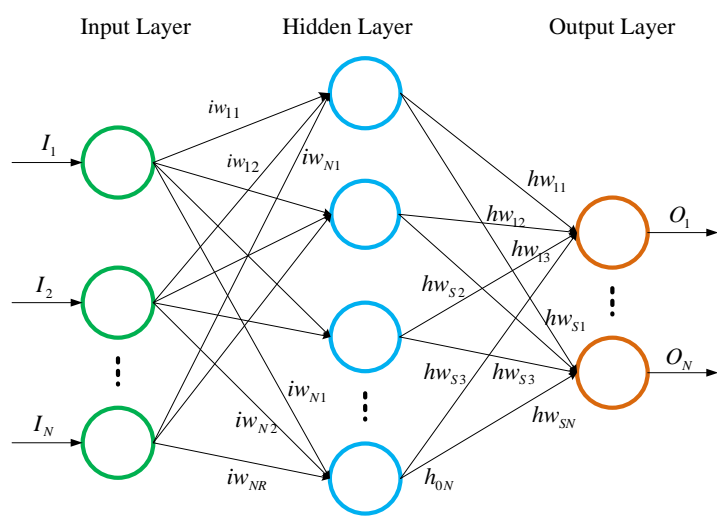

Fig.2. Internal structure of the NN

Likewise, on different neurons transfer function might contrast (normally were similar). ANN training necessary for make easy correct network arrangement. In many methods of ASN training, the very common method is backpropagation. To the modification of weight the Backpropagation technique is a slope type adjustment and can take more periods for training the network [36]. Here we recommend the ALO use as a training algorithm.

\section{Training Process of ANN with ALO}

Step 1: Read in input and outputs are expected

Step 2: Calculate the result through sum of weighted on inputs as well as by passing transfer functions

Step 3: Compare the result along desired result

Step 4: Calculate and update the fitness value in terms of comparison.

Step 5: The steps repeat 2 and 3 till completed the entire training points using the ALO algorithm

Step 6: To improve the fitness the weight is adjusted in the suitable direction

Step 7: The steps repeat 1-6 till identified the acceptable fitness value.

Every neuron is related along the transfer function, which performs of input. The neuron's input is the sum of weights on its inputs. The equation (10) is calculated as total weight of inputs,

$t_{j}=\sum_{i=1}^{n}\left(W_{i j}, Y_{i}\right)-B_{j} ; j=1,2, \ldots, h$

Here, $n$ specifies various input nodes, $W_{i j}$ specifies weight organization from $i^{\text {th }}$ node at layer of input for $j^{\text {th }}$ node at layer of hidden, $Y_{i}$ indicates the $i^{\text {th }}$ input, $h$ is the various hidden nodes, and $B_{j}$ indicates bias (threshold) on $j^{\text {th }}$ nodes of hidden. Sigmoid function is a better transfer function [37]. The sigmoid function assigns the input to the range $[0,1]$, which is described in equation (11),

$\operatorname{sigmoid}\left(t_{j}\right)=T_{j}=\frac{1}{1+e^{-t_{j}}} ; j=1,2, \ldots, h$

At last output is classified according to calculated nodes of hidden output, which is illustrated as equation (12) and (13), 
$F_{k}=\operatorname{sigmoid}\left(f_{k}\right)=1 / 1+e^{-f_{k}} ; k=1,2, \ldots, m$

$$
f_{k}=\sum_{j=1}^{h}\left(W_{j k}, T_{i}\right)-B_{k}^{\prime} ; k=1,2, \ldots, m
$$

Here, $W_{j k}$ represent weight connection from hidden node $j^{\text {th }}$ to output node $k^{\text {th }}, m$ represent various outputs, as well as $B_{k}^{\prime}$ represent threshold output node of $k^{\text {th }}$.

ANN output is clear from the above three steps is determined by weights and biases [38]. Here, the ALO algorithm is used as a trainer to parameters of ANN. The algorithm of ALO is applied for training process that is illustrated given below.

\section{2) The ALO Algorithm for Selecting Optimal Training Dataset}

Mirjalili in 2015 [39] have been presented the new natureinspired ALO algorithm which simulates in the trap linked among the ants and ant-lions. ALO represents linked among traps of ant as well as ant lion. Ants needed for shifting over the search area to model such interactions allowing ant lions to hunt and filter using traps. The five important steps are hunting are (1) agent's random walk (2) traps of building (3) ants in trap entrapment (4) prey of catching and (5) traps rebuilding. In ALO optimizer, wheel roulette as well as ants random walk may remove local optima [40]. The ants are moving to search food randomly. The following equation (14) is expressed by ants' random movement.

$X(t)=\left[\begin{array}{l}0, \\ \operatorname{cumsum}\left(2 r\left(t_{1}\right)-1\right), \\ \operatorname{cumsum}\left(2 r\left(t_{2}\right)-1\right), \ldots \ldots . \\ \operatorname{cumsum}\left(2 r\left(t_{n}\right)-1\right)\end{array}\right]$

Here, cumulative sum denoted as cumsum, $n$ signifies as ant's maximum number, $t$ described as random walk (iteration) step and $r(t)$ denotes the random number generation, rand specify random number created along uniform distribution in $[0,1]$, which given as equation (15),

$r(t)= \begin{cases}1 & \text { if rand }>0.5 \\ 0 & \text { if rand } \leq 0.5\end{cases}$

Among the search space for controlling random walk, using normalizing form, min-max normalization based. Ant's position can be updated using equation (16),

$X_{m}^{t}=\frac{\left(X_{m}^{t}-a_{m}\right)\left(d_{m}-c_{m}^{t}\right)}{d_{m}^{t}-a_{m}}+c_{t}$

Here, $a_{m}$ and $b_{m}$ are ants random walk's minimum and maximum, $c_{m}^{t}$ and $d_{m}^{t}$ denotes in $t^{\text {th }}$ iteration minimum and maximum variable of $m^{\text {th }}$. The random positions on ants are saved at matrix $M_{a n t}$ is given as equation (17),
$M_{\text {ant }}=\left(\begin{array}{cccc}A_{1,1} & A_{1,2} & \ldots & A_{1, n} \\ A_{2,1} & A_{2,2} & \ldots & A_{2, d} \\ \vdots & \vdots & \vdots & \vdots \\ A_{n, 1} & A_{n, 2} & \ldots & A_{n, d}\end{array}\right)$

Here, $A_{m, n}$ is $m^{\text {th }}$ variable value (dimension) of $n^{\text {th }}$ ant, $n$ denotes the many ants (population size). Total ants fitness is saved at matrix $M_{O A}$ based objective function $f$ is given as equation (18),

$M_{O A}=\left(\begin{array}{c}f\left(\left[A_{1,1}, A_{1,2}, \ldots, A_{1, n}\right]\right) \\ f\left(\left[A_{2,1}, A_{2,2}, \ldots, A_{2, d}\right]\right) \\ \vdots \\ f\left(\left[A_{n, 1}, A_{n, 2}, \ldots, A_{n, d}\right]\right)\end{array}\right)$

The ant-lion's position and fitness are specified through matrices $M_{\text {antlion }}$ and $M_{O A L}$, which is given as equation (19) and (20),

$$
\begin{gathered}
M_{\text {antlion }}=\left(\begin{array}{c}
A L_{1,1}, A L_{1,2}, \ldots, A L_{1, n} \\
A L_{2,1}, A L_{2,2}, \ldots, A L_{2, d} \\
\vdots \\
A L_{n, 1}, A L_{n, 2}, \ldots, A L_{n, d}
\end{array}\right) \\
M_{O A L}=\left(\begin{array}{c}
f\left(\left[A L_{1,1}, A L_{1,2}, \ldots, A L_{1, n}\right]\right) \\
f\left(\left[A L_{2,1}, A L_{2,2}, \ldots, A L_{2, d}\right]\right) \\
\vdots \\
f\left(\left[A L_{n, 1}, A L_{n, 2}, \ldots, A L_{n, d}\right]\right)
\end{array}\right)
\end{gathered}
$$

Ant's random walk is damaged through traps of ant lions. The expression of ants trapping to the pits of ant lion's,

$c_{m}^{t}=A n t-\operatorname{lion}_{n}^{t}+c^{t}$

$d_{m}^{t}=A n t-\operatorname{lion}_{n}^{t}+d^{t}$

The suitable ant-lion is chosen utilizing wheel roulette method. To choose ant lions in terms of their fitness through the iteration the ALO algorithm requires roulette wheel operator use. The process shows more chances of catching ants [41]. Along before methods, ant lions could form traps associated with fitness and ants must randomly walk. Further, perceive once there is an ant in trap ant lions should sands outside the pit center. Leaning down an ant, which is effort to get away this conduct. The ant's random style decreased with radius of the hyper sphere to model this conduct. Equations (23) and (24) represent sliding of ants into pits

$c^{t}=\frac{c^{t}}{I}$ 
$d^{t}=\frac{d^{t}}{I}$

Here, $I=10^{w} \frac{t}{S}$, current iteration specifies $t$, maximum number of iterations specifies $S$ and constant whose value represents $W$. The objective function is computed in this step. If ant having best objective function to choosen ant lion it will alter the stage for hunted ant's recent stage for improving this opportunity on capturing new one. The ant position must be updated to catch new prey after this [42]. It is specified in equation (25),

Ant lion $_{n}^{t}=$ Ant $_{m}^{t}$ if $f\left(\right.$ Ant $\left._{m}^{t}\right)>f\left(\right.$ Ant lion $\left._{j}^{t}\right)$

To keep better results in each stage the elitism is utilized. The better ant lion received is considered the elite, and is the fittest ant lion.

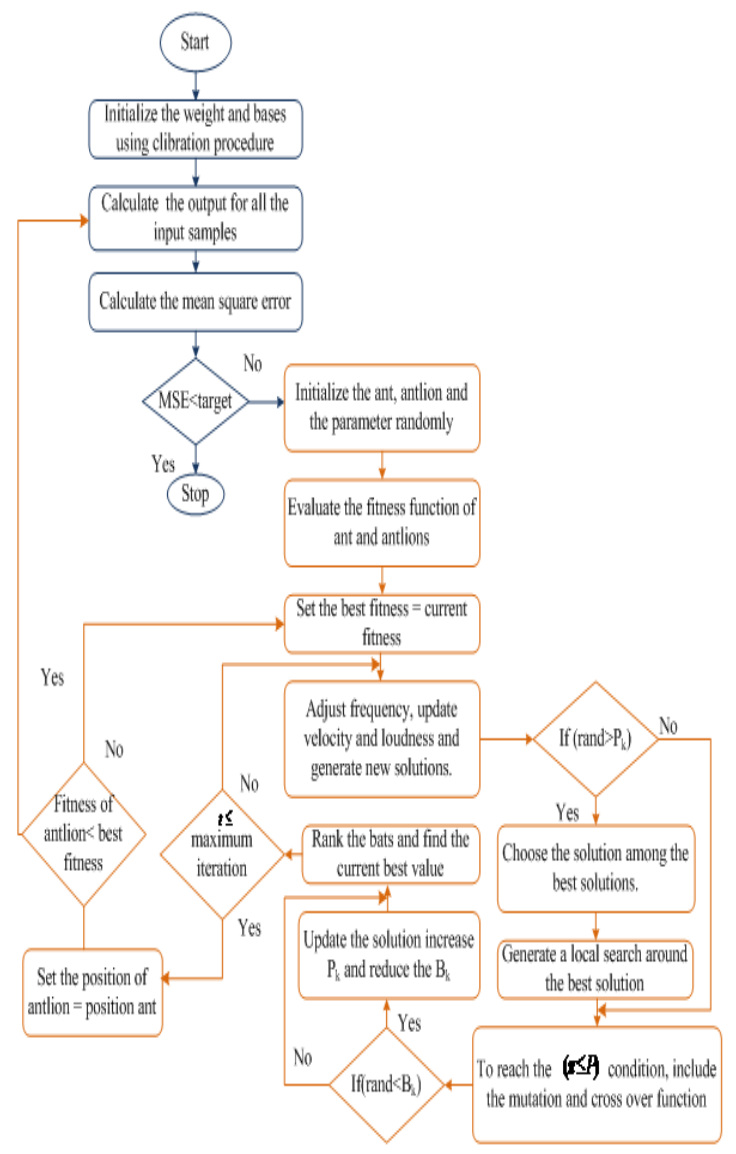

Figure3. Flow diagram of the proposed technique

The elite must infect at each level the ant lion (random movement). So that, each ant thought to be related along ant lion through elite and roulette wheel, provided through the equation (26),

$A n t^{t}=\frac{R_{A}^{t}+R_{B}^{t}}{2}$

Here, $R_{A}, R_{B}$ specify the selected ant lion of random walk around as well as elite in $t^{\text {th }}$ iteration at same time.
The ant's random walk is often prohibited around elite ant lions and as a result, algorithm faces bad research. It is a popular reality that effectiveness of meta-heuristic algorithms depends on the correct balance among exploration and exploitation.

Complete the process of neural network training, the network is well-trained to gathering optimal gain values of the PID controller at any instant. The proposed technique flow diagram is illustrated in figure 3 . To verify the optimal training dataset and the best solution is preferred, after getting the optimal solution the process is terminated otherwise it will continue the training process.

\section{RESULTS AND DISCUSSIONS}

We discuss presented method on high-order TDS with PID controller and tuning performance comparing with previous techniques like ABC, GSA as well as FA technique. The presented model is improved and executed at MATLAB 7.10.0 (R2015a) work site. The implementation process is using the types of dissimilar of higher order TDS along PID controller as well as various system model. Finally the presented technique performance is higher than that of other techniques that can be assessed in comparison with other techniques.

Table1: Parameters of implementation and values.

\begin{tabular}{cc}
\hline Parameters & Values \\
\hline Time constant $(\mathrm{T})$ & $360 \mathrm{sec}$ \\
Time delay $(\theta)$ & 1 \\
Steady state gain $(\mathrm{K})$ & 14.9 \\
Reference step $(\mathrm{r})$ & 10 \\
Simulation time $\left(\mathrm{T}_{\mathrm{f}}\right)$ & $1000 \mathrm{sec}$ \\
\hline
\end{tabular}

Table 1 tabulated needed parameters of implementation. There are five higher order TDS are utilized to presented method presentation and TDS are defined by given equation (27), (28), (29) and (30).

System 1: $\frac{1}{(s+1)(s+5)^{2}} e^{-0.5 s}$

System2: $\frac{1}{\left(s^{2}+2 s+3\right)(s+3)} e^{-0.3 s}$

System 3: $\frac{(1.2 s+1)^{2}}{(5 s+1)(s+1)^{2}} e^{-0.5 s}$

System 4: $\frac{20(50 s+1)}{1250 s^{2}+100 s+1} e^{-s}$

The presented system analysis of performance is defined along various computer models the simulation time is taken as 1000 seconds. The high-order TDS taken as delay of time and the PID controller regulated through presented technique with high-order TDS.

Published By:

Blue Eyes Intelligence Engineering 
For analyzing system performance output on system models can be determined with high-order TDS along some previous technique. The PID controller analysis of performance examines the following diagram.

The presented method model analysis of performance with some various techniques is presented in figure 4 . The settling time, rise time, peak overshoot time are evaluated depend on the response steps tabulated in table1. Depend on the comparison the proposed technique has rise time is 83 sec, settling time is $445 \mathrm{sec}$, overshoot is $95 \mathrm{sec}$ and the peak time is 109 sec. The peak value is 10.48 , which less overshoot and no under shoot from the proposed technique.

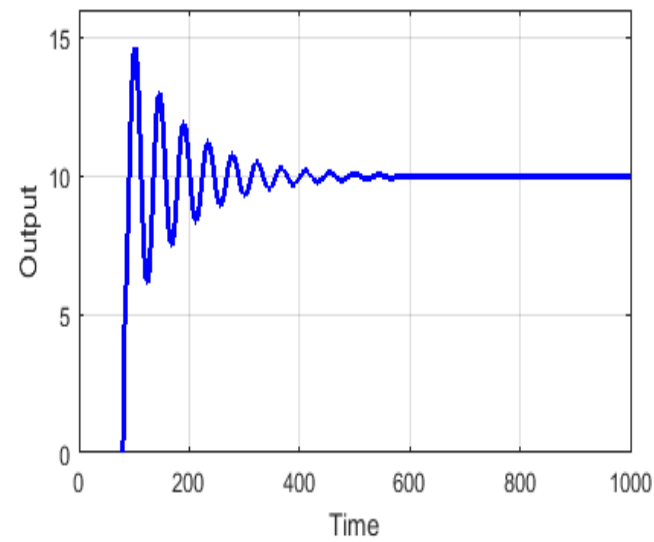

(i)

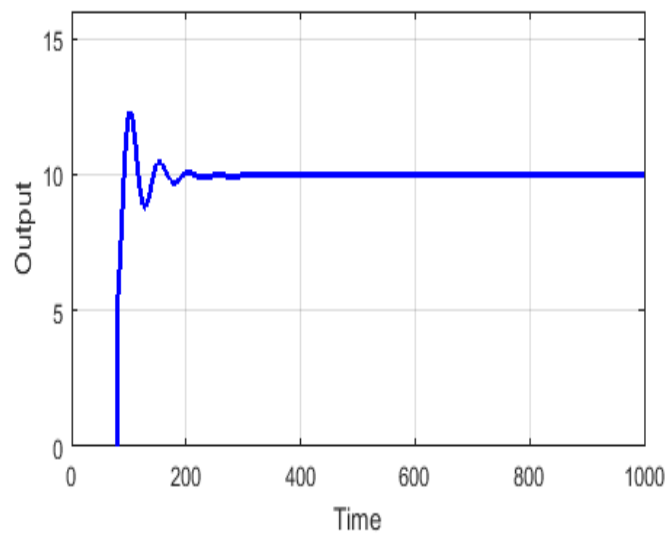

(ii)

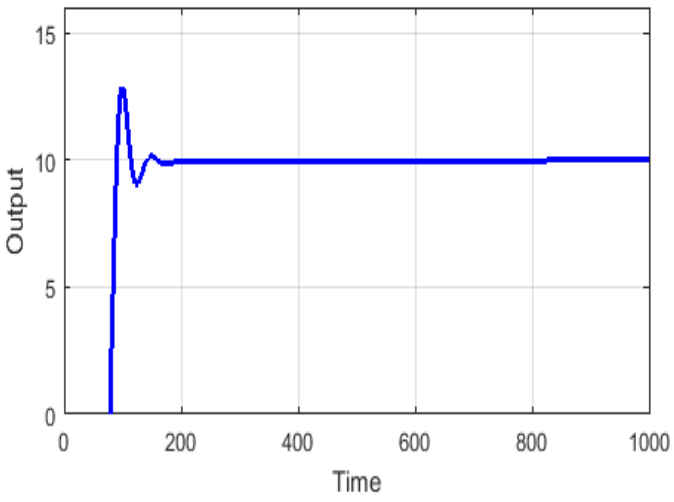

(iii)

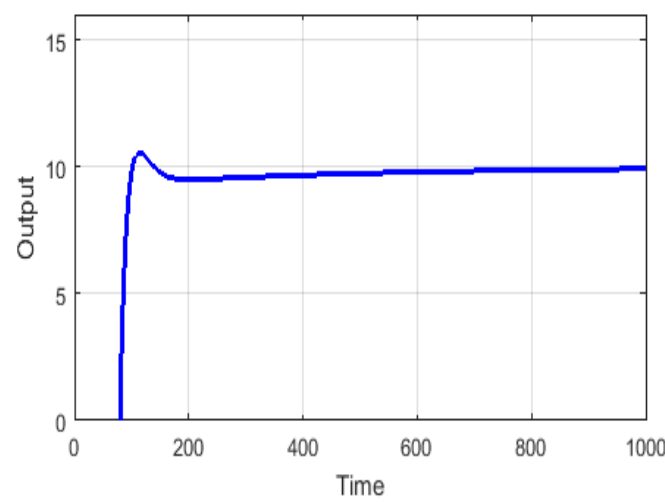

(iv)

Figure4. High-order TDS step response (i) FA, (ii) GSA, (iii) ABC and (iv) Proposed

Table 2 : The time domain specifications of the higher order time delay system

\begin{tabular}{ccccccc}
\hline $\begin{array}{c}\text { Algori } \\
\text { thm }\end{array}$ & $\begin{array}{c}\text { Rise } \\
\text { Time }\end{array}$ & $\begin{array}{c}\text { Settling } \\
\text { Time }\end{array}$ & $\begin{array}{c}\text { Overs } \\
\text { hoot }\end{array}$ & $\begin{array}{c}\text { Pe } \\
\text { ak }\end{array}$ & $\begin{array}{c}\text { Peak } \\
\text { Time }\end{array}$ & $\begin{array}{c}\text { Un } \\
\text { der } \\
\text { sho } \\
\text { ot }\end{array}$ \\
\hline $\begin{array}{c}\text { Propo } \\
\text { sed }\end{array}$ & 83 & 445 & 95 & $\begin{array}{c}10 . \\
48\end{array}$ & 109 & 0 \\
ABC & 84 & 510 & 97 & 12. & 99 & 121 \\
GSA & 89 & 560 & 120 & 13 & 110 & 122 \\
FA & 102 & 793 & 102 & 14. & 115 & 123 \\
\hline
\end{tabular}

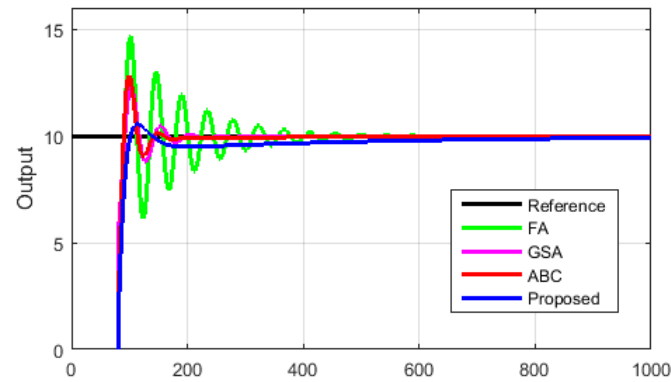

(a)

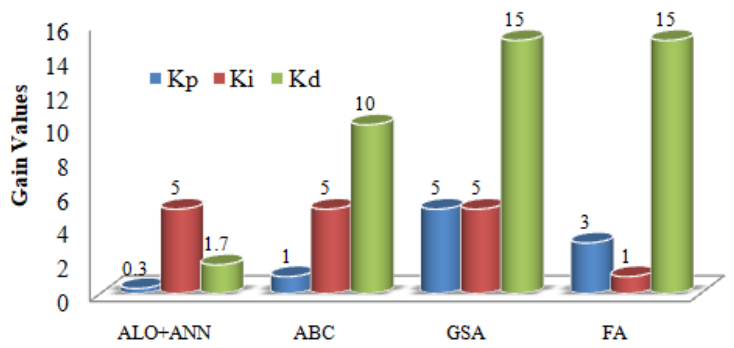

(b) 


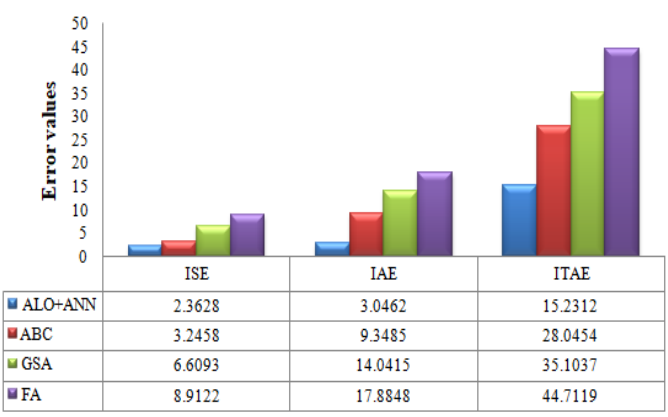

(c)

Fig.5. High-order system comparison analysis (a) performance, (b) gain values and (c) error values with various methods

High-order system comparison analysis comparison is shown in figure 5. Step response gain parameters and illustrated the Performance Indices (PIs) such as ISE, IAE and ITAE with some different techniques. The comparison of PIs between various methods of tuning consider PID controller to control response while used to high-order TDS. In different controllers, the PID controller structured along the proposed technique provides a particular reduction in error profiling for all interrupts. In step response, compared to the maximum error the ISE, IAE and ITAE are reduced.

\section{CONCLUSION}

Hence the proposed technique was utilized to adjust the PID controller with high-order TDS. The combination of ALO and ANN algorithms were presented technique, the ALO were used to develop the learning process of ANN in terms of the optimal training dataset. For control parameter of time delay system with optimal values, developed the performance. The time domain coefficients depends upon the higher order system output parameter variations. The proposed methodology was implemented with different high-order TDS and domain of time specifications like settling time, rise time, peak overshoot time is examined. The effectiveness of presented technique was determined using the comparative analysis with the existing control techniques. The comparative analysis proved that the presented method was well effective method for tuning the high-order TDS for different non-linear system model.

\section{REFERENCES}

1. A.A.Khandekar, G.M.Malwatkar and B.M.Patre, "Discrete sliding mode control for robust tracking of higher order delay time systems with experimental application", ISA Transactions, Vol.52, 2013, pp.36-44

2. S.P.Agnihotri and L. M.Waghmare, "Regression model for tuning the PID controller with fractional order time delay system", Ain ShamsEngineering Journal, Vol.5, No.4, pp.1071-1081, 2014

3. E.R.Samuel, L.Knockaert and T.Dhaene, "Model Order Reduction of Time-Delay Systems Using a Laguerre Expansion Technique," IEEE Transactions on Circuits and Systems, Vol.61, 2014, pp.1815-1823

4. G.M.Tamilselvan and P.Aarthy, "Online tuning of fuzzy logic controller using Kalman algorithm for conical tank system", An International Journal of Applied Research and Technology, 2017

5. V. Bobal, M.Kubalcik, P. Dostal and J.Matejicek, "Adaptive predictive control of time-delay systems", An International Journal of Computers and Mathematics with Applications, Vol.66, 2013,pp.165-176

6. Y. Cheon, K .H Ryu, S.W Sung, J. Lee and I.B Lee, "PID auto-tuning using new model reduction method and explicit PID tuning rule for a fractional order plus time delay model", An International Journal of Process Control, Vol.24, 2014, pp.113-128

7. M. M. Sabir and T. Ali, "Optimal PID controller design through swarm intelligence algorithms for sun tracking system", An International Journal of Applied Mathematics and Computation, Vol.274, 2016, pp.690-699

8. K.A.Gopala Rao, B.Amarendra Reddy and P. DurgaBhavani, "Fuzzy Pi and Integrating Type Fuzzy PID Controllersof Linear, Nonlinear and Time -Delay Systems", An International Journal of Computer Applications, Vol.1, 2010, pp.41-47

9. J.W. Perng, G.Y. Chen and Y. W. Hsu, "FOPID controller optimization based on SIWPSO-RBFNN algorithm for fractional-order time delay systems", An International Journal of Soft Computing, Vol.21, 2017, pp.4005-4018

10. D. Fister, I. Fister and R.Safaric, "Parameter tuning of PID controller with reactive nature-inspired algorithms", An International Journal of Robotics and Autonomous Systems, Vol.84, pp.64-75, 2016

11. A. Mesbahi and M.Haeri, "Robust non-fragile fractional order PID controller for linear time invariant fractional delay systems", An International Journal of Process Control, Vol.24, 2014, pp.1489-1494

12. D. Li, L. Liu, Q. Jin and K.Hirasawa, "Maximum sensitivity based fractional IMC-PID controller design for non-integer order system with time delay", An International Journal of Process Control, Vol.31, 2015, pp.17-29,

13. H. Liu, Y. Li, Y. Zhang, Y. Chen, Z. Song, Z. Wang, S. Zhang and J. Qian, "Intelligent Tuning Method of PID Parameters Based on Iterative Learning Control for Atomic Force Microscopy", An International Journal of Micron, Vol.104, 2018, pp.26-36

14. A.Moharam, Mostafa A.El-Hosseini and Hesham A.Ali, "Design of optimal PID controller using hybrid differential evolution and particle swarm optimization with an aging leader and challengers", An International Journal of Applied Soft Computing, Vol.38, 2016, pp.727-737

15. X. Gao, C. Shang, D. Huang and F. Yang, "A novel approach to monitoring and maintenance of industrial PID controllers", An International Journal of Control Engineering Practice, Vol.64, 2017, pp.111-126

16. B. Hamed and W.Issa, "A Modified Internal Model Control for Unstable-Time Delayed System", An International Journal of Engineering and Advanced Technology (IJEAT), Vol.1, 2011, pp.5662 ,

17. D.Kumanan and B.Nagaraj, "Tuning of proportional integral derivative controller based on firefly algorithm", An Open Access Journal of Systems Science \& Control Engineering, Vol.1, 2013, pp.52-56

18. B. Bhushan and M. Singh, "Adaptive control of DC motor using bacterial foraging algorithm", An International Journal of Applied Soft Computing, Vol.11, 2011, pp.4913-4920

19. I. Chiha, N.Liouane and Pierre Borne, "Tuning PID Controller Using Multiobjective Ant Colony Optimization", An International Journal of Applied Computational Intelligence and Soft Computing, Vol.2012, 2012, pp.1-7

20. S.J.Suji Prasad, Susan Varghese and P.A.Balakrishnan, "Particle Swarm Optimized I-PD Controller for Second Order Time Delayed System", An International Journal of Soft Computing and Engineering (IJSCE), Vol.2, No.1, pp.229-302, 2012

21. K.Ghousiya Begum, A.Seshagiri Rao and T.K.Radhakrishnan, "Enhanced IMC based PID controller design for non-minimum phase (NMP) integrating processes with time delays", ISA Transactions, Vol.68, 2017, pp.223-234

22. P.Prommee and K. Angkeaw, "High performance electronically tunable log-domain current-mode PID controller", An International Journal of Microelectronics,2017, pp.1-12

23. Q.H. Seer and J.Nandong, "Stabilization and PID tuning algorithms for second-order unstable processes with time-delays", ISA Transactions, Vol.67, 2017, pp.233-245

24. A. Kumar and V. Kumar, "A novel interval type-2 fractional order fuzzy PID controller: Design, performance evaluation, and its optimal time domain tuning", ISA Transactions, Vol.68, 2017, pp.251-275

25. H.S. Sanchez, A.Visioli and R.Vilanova, "Optimal Nash tuning rules for robust PID controllers", An International Journal of the Franklin Institute, Vol.354, 2017, pp.3945-3970

26. J. Mendes and L. Osorio and R.Araujo, "Self-tuning PID controllers in pursuit of plug and play capacity", An International Journal of Control Engineering Practice, Vol.69, 2017, pp.73-84

27. D. Lee, Y. Cheon, J.H. Ryu and I.B. Lee, "An MCFC operation optimization strategy based on PID auto-tuning control", An International Journal ofHydrogenEnergy, Vol.42, 2017, pp.2551825530 
28. X. Luan, Q. Chen, P. Albertos and F. Liu, "Conversion of SISO processes with multiple time-delays to single time-delay processes", An International Journal of Process Control, 2017

29. Q. Chai and W. Wang, "A computational method for free terminal time optimal control problem governed by nonlinear time delayed systems", An International Journal of Applied Mathematical Modelling, Vol.53, 2018, pp.242-250

30. R.Sen, C.Pati, S.Dutta and R.Sen, "Comparison Between Three Tuning Methods of PID Control for High Precision Positioning Stage", MAPAN-Journal of Metrology Society of India, Vol.30, No.1, pp.6570, 2015

31. S.N.Deepa and G.Sugumaran, "Design of PID Controller for Higher Order Continuous Systems using MPSO based Model Formulation Technique", An International Journal of Computer, Electrical, Automation, Control and Information Engineering Vol.5, 2011

32. S. Srivastava, A.Misra, S.K.Thakur and V.S.Pandit, "An optimal PID controller via LQR for standard second order plus time delay systems", ISA Transactions, Vol.60, 22016, pp.244-253

33. S. Saxena and YV.Hote, "Simple Approach to Design PID Controller via Internal Model Control", Arabian Journal for Science and Engineering, Vol.41, 2016, pp.3473-3489

34. C..Anil and R.P.Sree, "Tuning of PID controllers for integrating systems using direct synthesis method", ISA Transactions, Vol.57, 2015pp.211-219

35. A. Askarzadeh and A.Rezazadeh, "Artificial neural network training using a new efficient optimization algorithm", An International Journal of Applied Soft Computing, Vol.13, 2013, pp.1206-1213

36. E. Deniz, O. Aydogmus and Z.Aydogmus, "Implementation of ANNbased Selective Harmonic Elimination PWM using Hybrid Genetic Algorithm-based optimization", An International Journal of Measurement, Vol.85, 2016, pp.32-42

37. G. Das, P.K. Pattnaik and S. K.Padhy, "Artificial Neural Network trained by Particle Swarm Optimization for non-linear channel equalization", An International Journal of Expert Systems with Applications, Vol.41, 2014, pp.3491-3496

38. W. Yamany, A. Tharwat, M. Fawzy, T. Gaber and A. E. Hassanien, "A New Multi-layer Perceptrons Trainer based on Ant Lion Optimization Algorithm", In proceedings of Fourth International Conference on Information Science and Industrial Applications (ISI), Busan,2015, pp.40-45

39. S. Mirjalili, "The Ant Lion Optimizer", An International Journal of Advances in Engineering Software, Vol.83, 2015, pp.80-98

40. P. DinakaraPrasasd Reddy, V.C Veera Reddy and T.GowriManohar, "Ant Lion optimization algorithm for optimal sizing of renewable resources for loss reduction in distribution systems", An International Journal of Electrical Systems and Information Technology, 2017

41. N.Samon, Z.M.Yasin and Z.Zakaria, "Ant Lion Optimizer for Solving Unit Commitment Problem", An International Journal of Advances in Electronics and Computer Science, Vol.4, 2017, pp.3-7

42. E.S.Ali, S.M.Abd Elazim and A.Y.Abdelaziz, "Ant Lion Optimization Algorithm for optimal location and sizing of renewable distributed generations", An International Journal of Renewable Energy, Vol.101, 2017, pp.1311-1324 